\title{
Prospective Associations Between Socioeconomically Disadvantaged Groups and Metabolic Syndrome Risk in European Children Results from the IDEFICS Study
}

Iguacel, Isabel; Michels, Nathalie; Ahrens, Wolfgang; Bammann, Karin; Eiben, Gabriele; Fernandez-Alvira, Juan M.; Mårild, Staffan; Molnar, Dénes; Reisch, Lucia A.; Russo, Paola; Tornaritis, Michael; Veidebaum, Toomas; Wolters, Maike; Moreno, Luis Alberto; Börnhorst, Claudia

Document Version

Accepted author manuscript

Published in:

International Journal of Cardiology

DOI:

10.1016/j.jijcard.2018.07.053

Publication date:

2018

License

CC BY-NC-ND

Citation for published version (APA):

Iguacel, I., Michels, N., Ahrens, W., Bammann, K., Eiben, G., Fernandez-Alvira, J. M., Mårild, S., Molnar, D., Reisch, L. A., Russo, P., Tornaritis, M., Veidebaum, T., Wolters, M., Moreno, L. A., \& Börnhorst, C. (2018). Prospective Associations Between Socioeconomically Disadvantaged Groups and Metabolic Syndrome Risk in European Children: Results from the IDEFICS Study. International Journal of Cardiology, 272, 333-340. https://doi.org/10.1016/j.ijcard.2018.07.053

Link to publication in CBS Research Portal

\section{General rights}

Copyright and moral rights for the publications made accessible in the public portal are retained by the authors and/or other copyright owners and it is a condition of accessing publications that users recognise and abide by the legal requirements associated with these rights.

\section{Take down policy}

If you believe that this document breaches copyright please contact us (research.lib@cbs.dk) providing details, and we will remove access to the work immediately and investigate your claim. 


\section{Prospective Associations Between Socioeconomically Disadvantaged Groups and Metabolic Syndrome Risk in European Children: Results from the IDEFICS Study}

Isabel Iguacel, Nathalie Michels, Wolfgang Ahrens, Karin Bammann, Gabriele Eiben, Juan M. FernandezAlvira, Staffan Mårild, Dénes Molnar, Lucia A. Reisch, Paola Russo, Michael Tornaritis, Toomas Veidebaum, Maike Wolters, Luis Alberto Moreno, and Claudia Börnhorst

Journal article (Accepted manuscript*)

\section{Please cite this article as:}

Iguacel, I., Michels, N., Ahrens, W., Bammann, K., Eiben, G., Fernandez-Alvira, J. M., ... Börnhorst, C. (२०18). Prospective Associations Between Socioeconomically Disadvantaged Groups and Metabolic Syndrome Risk in European Children: Results from the IDEFICS Study. International Journal of Cardiology, 272, 333-340. D0I:

10.1016/j.jijcard.2018.07.053

DOI: 10.1016/j.jijcard.2018.07.053

* This version of the article has been accepted for publication and undergone full peer review but has not been through the copyediting, typesetting, pagination and proofreading process, which may lead to differences between this version and the publisher's final version AKA Version of Record.

Uploaded to CBS Research Portal: July २०19

(C) 2019. This manuscript version is made available under the CL-BY-NC-ND 4.0 license http://creativecommons.org/licenses/by-nc-nd/4.0/ 


\section{Accepted Manuscript}

Prospective associations between socioeconomically disadvantaged groups and metabolic syndrome risk in European children. Results from the IDEFICS study

Isabel Iguacel, Nathalie Michels, Wolfgang Ahrens, Karin Bammann, Gabriele Eiben, Juan M. Fernández-Alvira, Staffan Mårild, Dénes Molnár, Lucia Reisch, Paola Russo, Michael Tornaritis, Toomas Veidebaum, Maike Wolters, Luis A. Moreno, Claudia Börnhorst, on behalf of the IDEFICS consortium

PII:

S0167-5273(18)31889-8

DOI: doi:10.1016/j.ijcard.2018.07.053

Reference: IJCA 26720

To appear in: International Journal of Cardiology

Received date: 20 March 2018

Revised date: 28 June 2018

Accepted date:

9 July 2018

Please cite this article as: Isabel Iguacel, Nathalie Michels, Wolfgang Ahrens, Karin Bammann, Gabriele Eiben, Juan M. Fernández-Alvira, Staffan Mårild, Dénes Molnár, Lucia Reisch, Paola Russo, Michael Tornaritis, Toomas Veidebaum, Maike Wolters, Luis A. Moreno, Claudia Börnhorst, on behalf of the IDEFICS consortium , Prospective associations between socioeconomically disadvantaged groups and metabolic syndrome risk in European children. Results from the IDEFICS study. Ijca (2018), doi:10.1016/ j.ijcard.2018.07.053

This is a PDF file of an unedited manuscript that has been accepted for publication. As a service to our customers we are providing this early version of the manuscript. The manuscript will undergo copyediting, typesetting, and review of the resulting proof before it is published in its final form. Please note that during the production process errors may be discovered which could affect the content, and all legal disclaimers that apply to the journal pertain. 
PROSPECTIVE ASSOCIATIONS BETWEEN SOCIOECONOMICALLY DISADVANTAGED

GROUPS AND METABOLIC SYNDROME RISK IN EUROPEAN CHILDREN. RESULTS FROM THE IDEFICS STUDY.

Isabel Iguacel ${ }^{1,2,3,4+*}$, Nathalie Michels ${ }^{5}$, Wolfgang Ahrens ${ }^{6,7}$, Karin Bammann $^{6,8}$, Gabriele Eiben ${ }^{9,10}$, Juan M. Fernández-Alvira, ${ }^{1,11}$, Staffan Mårild ${ }^{9}$, Dénes Molnár ${ }^{12}$, Lucia Reisch ${ }^{13}$, Paola Russo ${ }^{14}$, Michael Tornaritis $^{15}$, Toomas Veidebaum ${ }^{16}$, Maike Wolters $^{6}$, Luis A. Moreno ${ }^{1,2,3,4,17}$, Claudia Börnhorst ${ }^{6,17}$, on behalf of the IDEFICS consortium

${ }^{1}$ GENUD (Growth, Exercise, NUtrition and Development) Research Group, Faculty of Health Sciences; University of Zaragoza, Edificio del SAI, C/Pedro Cerbuna s/n, 50009, Zaragoza, Spain

${ }^{2}$ Instituto Agroalimentario de Aragón (IA2), Zaragoza, Spain

${ }^{3}$ Instituto de Investigación Sanitaria Aragón (IIS Aragón), Zaragoza, Spain

${ }^{4}$ Centro de Investigación Biomédica en Red de Fisiopatología de la Obesidad y Nutrición (CIBERObn),

Zaragoza, Spain

${ }^{5}$ Department of Public Health, Ghent University, Ghent, Belgium

${ }^{6}$ Leibniz Institute for Prevention Research and Epidemiology - BIPS, Bremen, Germany

${ }^{7}$ Institute of Statistics, University of Bremen, Bremen, Germany

${ }^{8}$ Institute for Public Health and Nursing Sciences (IPP), University of Bremen, Bremen, Germany

${ }^{9}$ Section for Epidemiology and Social Medicine (EPSO), Sahlgrenska Academy, University of Gothenburg, Gothenburg, Sweden

${ }^{10}$ Department of Biomedicine and Public Health, School of Health and Education, University of Skövde,

Skövde, Sweden

${ }^{11}$ Fundación Centro Nacional de Investigaciones Cardiovasculares Carlos III (CNIC), Madrid, Spain

${ }^{12}$ Department of Paediatrics, University of Pécs, Pécs, Hungary

${ }^{13}$ Copenhagen Business School, Copenhagen, Denmark

${ }^{14}$ Institute of Food Sciences, National Research Council, Avellino, Italy

${ }^{15}$ Research and Education Institute of Child Health, Strovolos, Cyprus

${ }^{16}$ Department of Chronic Diseases, National Institute for Health Development, Tallinn, Estonia

${ }^{17}$ These authors contributed equally and share the last authorship. 
${ }^{+}$Isabel Iguacel takes responsibility for all aspects of the reliability and freedom from bias of the data presented and their discussed interpretation.

*Corresponding author: I. Iguacel, phone +34876553753 email iguacel@ unizar.es

Author contributions. This manuscript represents original work that has not been published previously and is currently not considered by another journal.

Individuals claiming authorship should meet all 3 of the following conditions:

1) Authors make substantial contributions to conception and design, and/or acquisition of data, and/or analysis and interpretation of data;

2) Authors participate in drafting the article or revising it critically for important intellectual content; and

3) Authors give final approval of the version to be submitted and any revised version.

Acknowledgments. This work was done as part of the IDEFICS study (www.idefics.eu). The authors wish to thank the IDEFICS children and their parents for participating in the extensive examination procedures involved in this study. The authors gratefully acknowledge the financial support of the European Commission within the Sixth RTD Framework Programme Contract No. 016181 (FOOD). I. I. has been financed by the FPU (grant reference FPU014/00922) Predoctoral Programs (Spanish Ministry of Education and Science)

Conflict of interest Statement. The authors declare that there are no conflicts of interest.

Keywords: metabolic syndrome, socioeconomic disadvantages, social vulnerabilities, obesity, children, migrants, lack of social support, family structure, socioeconomic status, modifiable lifestyle indicators. 


\section{Abstract}

OBJECTIVE Socioeconomic disadvantages during childhood are hypothesised to have negative implications for health. We aimed to investigate the association between socioeconomic disadvantages and children's total metabolic syndrome (MetS) score at baseline and follow-up and the extent to which socioeconomic disadvantages over time and the accumulation of these socioeconomic disadvantages can affect children's MetS risk.

METHODS The two-year longitudinal IDEFICS study included 2,401 European children (aged 2.0-9.9) with complete information of the 16,229 participating at baseline. Sociodemographic variables, psychosocial factors and lifestyle were proxy-reported via questionnaires. Socioeconomically disadvantaged groups included children from families with low income, low education, migrant origin, unemployed parents, parents who lacked a social network, and from non-traditional families. MetS risk score was calculated as the sum of $\mathrm{z}$-scores of waist circumference, blood pressure, lipids and insulin resistance. Linear mixed-effects models were used to study the association between social disadvantages and MetS risk. Models were adjusted for sex, age, well-being and lifestyle (fruit and vegetables consumption, physical activity, screen time).

RESULTS At both time points, children from low-income families (0.20 [0.03-0.37]); $\beta$ estimate and $99 \%$ confidence interval), children from non-traditional families (0.14 [0.02-0.26]), children whose parents were unemployed $(0.31[0.05-0.57])$ and children who accumulated more than 3 disadvantages (0.21 [0.04-0.37]) showed a higher MetS score compared to non-socioeconomically disadvantaged groups.

CONCLUSION Children from socioeconomically disadvantaged families are at high metabolic risk independently of diet, physical activity, sedentary behaviours and well-being. Interventions focusing on these socioeconomically disadvantaged groups should be developed to tackle health disparities. 


\section{Introduction}

Cardiovascular diseases are the most common cause of death in adults worldwide[1]. Risk factors for cardiovascular diseases and diabetes include abdominal obesity, hypertension, insulin resistance (IR), elevated triglycerides (TG) and reduced high-density lipoprotein cholesterol (HDL-C) tending to cluster as the metabolic syndrome (MetS)[2]. MetS affects over 24.3\% of European adults with a higher occurrence in Southern than in Northern Europe[3]. According to a study conducted in European children aged 2.0-10.9, prevalence of MetS was 5.5\%[4]. Although this percentage is lower in children than in adults, increasing rates of childhood obesity and sedentary lifestyle during the last decades could be associated with an increased prevalence of MetS in children and adolescents in the future[5].

Socioeconomic status (SES) is one of the main determinants of MetS in children and adults[6]. SES (often measured by determining education, occupation and income) is inversely associated with obesity and diabetes, both linked to MetS. Children and adults with low SES are more likely to have a poor diet, low levels of physical activity (PA) and high levels of sedentary behaviours and therefore, they show higher rates of obesity and diabetes than children and adults with high SES[7]. Higher SES in childhood has been associated with a lower risk for MetS more than 30 years later in adulthood independently of cardiometabolic risk factors in childhood and participants' SES in adulthood[8]. These results emphasize that obesity and MetS tracks into adulthood[9].

Despite some studies have analyzed the impact of low SES on MetS[6], there are other under-researched disadvantages in early life[10]. Additionally, most studies did not consider relevant information on mental health and lifestyle or focused on adults. Socioeconomic disadvantages are defined here as family and socioeconomic exposures negatively affecting children through behavioral, mental health and biological factors. Socioeconomic disadvantages in children have been linked with unhealthy lifestyles, higher levels of obesity and a poorer well-being compared to non-socioeconomically disadvantaged groups[1114].

In this study we used the general term socioeconomically disadvantaged groups, which included two low SES groups (children from families with low income and low education) and four social vulnerable 
groups (children whose parents were migrants, children whose parents lacked a social network, children from non-traditional families and children with unemployed parents).

Despite a vast literature has investigated the association between parental SES and children's lifestyle and obesity very few studies have studied the impact that parental socioeconomic disadvantages can have on children MetS risk. Prospective studies are needed to understand whether social disadvantages during childhood can have early consequences on metabolic parameters. Using longitudinal data these results would help to identify children at a higher risk of developing cardiovascular diseases in adulthood.

This prospective study aimed to investigate the association of 1) the six above-mentioned socioeconomic disadvantages and children's total MetS score at baseline and follow-up; 2) socioeconomic disadvantages over time and children's total MetS score at follow-up and 3) accumulation of these socioeconomic disadvantages and children's total MetS score at baseline and follow-up, in a large cohort of European children.

\section{Materials and methods}

\section{Study population}

The Identification and Prevention of Dietary-and Lifestyle-induced Health Effects in Children and Infants (IDEFICS) study is a multi-centre prospective cohort study including a school- and community-based obesity prevention intervention[15]. Eight pairs of mutually comparable areas were allocated in eight European countries (Belgium, Cyprus, Estonia, Germany, Hungary, Italy, Spain and Sweden) to either control status or intervention status (Table S1). For comprehensive information about IDEFICS, a detailed description is given in Ahrens et al.[16]. In the baseline survey (T0), 16,229 children aged 2.09.9 participated from September 2007 to June 2008. Follow-up (T1) took place two years later (September 2009-June 2010) applying same standardised assessments where 11,041 children aged 4.011.9 were re-examined. Parents or legal guardians gave written informed consent for examinations and data collection for their children, while children expressed oral consent. Ethical approval was obtained from the research ethics authority of each participating centre.

\section{Socioeconomically disadvantaged groups}

The following information was obtained from parental questionnaires.

Education: parents indicated their highest level of education. Response categories for each country were coded according to the International Standard Classification of Education (ISCED 1997) and re- 
categorised into three categories: low (ISCED level 0-2), medium (ISCED level 3-4) and high (ISCED level 5-6)[17]. The highest level of education of either the mother or the father was considered.

Income: parents provided information on household monthly net income responding to nine countryspecific categories (from lowest category, 1, to highest category, 9). Results were organised into three categories: low (1-3), medium (4-6) and high income (7-9).

Social network: a minimal social network was assumed if parental answer to 'How many persons, including your family, do you know that you can definitely rely on in cases of need?' was either 'Nobody' or ' 1 person'. A strong social network was defined when response was $\geq 2$ persons.

Family structure: children from 'Traditional families' lived with both biological parents and 'nontraditional families' included single-parent families, stepparent families, living with grandparents, other relatives or adults, foster parents or in an institution.

Origin of parents: a migrant background was assumed if one or both parents were born in a country different from where study took place.

Employment status: children with unemployed parents were those with either the mother or the father being unemployed or living on social assistance or welfare.

We calculated a total score by adding up the number of socioeconomic disadvantages a child was exposed to (low education, low income, minimal social network, non-traditional family, migrant, unemployed). Score ranged from 0-6 and was divided into four categories (three to six disadvantages, two disadvantages, one disadvantage and no disadvantages).

\section{Waist circumference}

Waist circumference (WC) $(\mathrm{cm})$ was measured in upright position with relaxed abdomen and feet together, midway between the lowest rib margin and the iliac crest to the nearest $0.1 \mathrm{~cm}$ with an inelastic tape (Seca 200).

\section{Blood pressure}

Blood pressure $(\mathrm{mmHg})$ was measured with an electronic sphygmomanometer (Welch Allyn 4200B-E2, USA). This electronic device has been validated and it records accurately blood pressure[18]. Before taking a blood pressure measurement, children were asked to sit for at least $5 \mathrm{~min}$. Two measurements were taken with 2 min interval in between. When first and second measurements differed by $>5 \%$ a third 
measurement was taken. For statistical analyses, the average of the two measurements was used. In case of three measurements, the average of the two measurements with the smallest difference was used.

\section{Blood collection for lipid and glucose homeostasis}

Depending on participants' preferences venepuncture or capillary sampling were used to collect fasting blood. A more detailed description about blood sampling procedures can be found in Ahrens et al[4]. Assessment of blood glucose, HDL-C and TG was performed on site at each study center within 5 min of blood withdrawal by placing one drop of blood in the 'point-of-care' analyzer Cholestech LDX (Cholestech, Hayward, CA, USA). Blood samples were analysed using a luminescence immunoassay (AUTO-GA Immulite 2000, Germany) for insulin ( $\mu \mathrm{IU} / \mathrm{ml}$ ). The homeostasis model assessment (HOMA)[19] was used as measure of IR with this formula: HOMA=fasting insulin $(\mu \mathrm{IU} / \mathrm{ml}) \times$ fasting glucose $(\mathrm{mg} / \mathrm{dl}) / 405$.

\section{Metabolic syndrome score}

MetS score, developed by Ahrens et al.[4], was calculated as the sum of sex- and age-specific z-scores of WC, HOMA-IR index, mean of z-scores of diastolic and systolic blood pressure (SBP, DBP) and mean of z-score of HDL cholesterol multiplied by -1 and z-score of triglycerides (TG). A higher score suggests a higher metabolic risk.

\section{Covariate information}

Child sex, age, country and information on children's well-being and lifestyle factors was collected using proxy-reported questionnaires during baseline survey.

\section{Children's well-being score}

A well-being score was calculated based on questions on emotional well-being, self-esteem, family relations and social contacts[20] adopted from KINDL®[14]. This score ranged from 12-48 with higher values indicating a better well-being[21].

\section{Lifestyle indicators}

Fruits and vegetables consumption was obtained using the food frequency section of Children's Eating Habits Questionnaire[22]. This questionnaire is a validated screening tool where parents reported usual 
at-home consumption frequencies of 42 food items of the previous four weeks. To have a healthy diet indicator we summed reported weekly intake frequencies of fresh fruits, raw and cooked vegetables.

Physical activity: Parents reported weekly hours children spent playing outdoors and participating in sports club activities in the previous month.

Screen time (ST): Parents reported daily ST their children spent on audio-visual media (TV, video, DVD, computer, game console) for a typical weekday and weekend day.

As respondents with missing socio-economic information may not be a random subset of populationbased survey participants and excluding records with missing income information from analyses may bias study results[23], missing values in socio-economic variables and vulnerability indicators were coded as a separate category.

After excluding children with missing values in MetS score at baseline or follow-up, well-being score and any lifestyle indicators at baseline, this analysis finally included 2,401 children (52.4\% boys) (Supplemental Figure S1).

\section{Statistical analyses}

For descriptive purposes, children were divided in two categories: at risk of MetS $\left(\geq 90^{\text {th }}\right.$ percentile of MetS score) versus not at risk $\left(<90^{\text {th }}\right.$ percentile of MetS score).

Linear mixed-effects models were used to assess cross-sectional and prospective associations between the six disadvantaged groups and MetS score. All models included a random kindergarten/school and a random country effect to account for the clustered study design.

One cross-sectional and three prospective analyses were conducted. For each exposure assessed at T0, a model was estimated to evaluate associations with children's MetS score at T0. In prospective analyses, we related 1) each exposure at T0 with children's MetS at T1; 2) socioeconomic disadvantages over time (disadvantaged at $\mathrm{T} 0$ and $\mathrm{T} 1$, disadvantaged at $\mathrm{T} 0$ and non-disadvantaged at $\mathrm{T} 1$, non-disadvantaged at $\mathrm{T} 0$ and disadvantaged at T1 and non-disadvantaged at T0 and T1) and children's MetS score at T1 and 3) accumulation of socioeconomic disadvantages at T0 and children's MetS score at T0 and T1. Since migrant status does not change between baseline and follow-up, patterns of socioeconomic disadvantages were investigated for the other disadvantaged groups only. 
All models were adjusted for baseline age, sex, children's well-being score and lifestyle factors (frequency of fruit and vegetable consumption, PA and total ST). Social vulnerabilities were also adjusted for parental education and income to assess the effect independent of classical SES indicators. For prospective analyses, a variable indicating intervention versus control region was added and models were additionally adjusted for baseline outcome values (children's MetS at T0).

Before model building, correlations among socioeconomic disadvantages were checked ranging from $r=0.01$ to $r=0.47$. The largest correlation was found between occupation and income $(r=0.47)$ such that parental occupation was not included in the models together with the other socioeconomic disadvantages to avoid collinearity problems.

Significance level was set at $\mathrm{p}<0.01$ to account at least partially for multiple testing. Analyses were performed using the Statistical Package for the Social Sciences (version 22.0; SPSS).

\section{Results}

Table 1 summarises distributions of predictors and covariates stratified by children's MetS risk at T0 and T1. At T1, older children, males, children with socioeconomic disadvantages or with missing information on socioeconomic disadvantages studied (except for parental education) had a higher percentage of MetS risk than their counterparts without socioeconomic disadvantages. Children at risk for MetS had lower fruit and vegetables consumption, lower well-being score and higher ST compared to those not at risk, while mean PA was lower at T1 only.

Table 2 presents $\beta$ estimates $(\beta), 99 \%$ confidence intervals (CI) and p-values for models assessing crosssectional and prospective associations between six socioeconomically disadvantaged groups and children's MetS score at baseline and follow-up, respectively. At T0, in models with basic adjustment, children from low-income families $(\beta 0.17$ [99\%CI 0.01-0.31]) and children from families with a low ( $\beta$ 0.28 [99\%CI 0.04-0.52]) and medium ( $\beta$ 0.17 [99\%CI 0.06-0.28]) parental education showed a significantly higher MetS score compared to the corresponding non-socioeconomically disadvantaged groups. In fully-adjusted models (additionally adjusted for well-being score and lifestyle factors) $\beta$ estimates were attenuated rendering them statistically insignificant (except for children from families with medium education).

Table 3 displays results for associations between patterns of socioeconomic disadvantages over time and children's MetS score at T1. In fully-adjusted models, children from non-traditional families at both time 
points $(\beta 0.14$ [99\% CI 0.02-0.26]) and children with unemployed parents at baseline and follow-up ( $\beta$ 0.31 [99\%CI 0.05-0.57]) had a significantly higher MetS score compared to children who were living with both biological parents and compared to children whose parents were employed at T0 and T1, respectively.

Despite the lack of statistical significance, results pointed to the expected direction for all socioeconomic disadvantages, i.e. MetS risk was higher in children showing disadvantaged patterns. A similar high risk of MetS at T1 was found in children with missing information on their family structure or parental social network compared to children of traditional families or strong social network parents.

Table 4 shows associations between accumulation of six socioeconomic disadvantages assessed at baseline and MetS score at T0 and T1. Children with 3 or more socioeconomic disadvantages accumulated had a significantly higher MetS score at T1 ( $\beta$ 0.21 [99\% CI 0.04-0.37]) compared to children with less socioeconomic disadvantages. Results at T0 pointed to the same direction though not reaching statistical significance.

\section{Sensitivity analyses}

Role of covariates:

Unadjusted models showed stronger associations between socioeconomic disadvantages on children's MetS score than fully adjusted models. Concerning the roles of covariates, $\beta$ estimates were attenuated when adding lifestyle factors and children's well-being. The greatest attenuation was found when adding ST to the models. Adjustment for classical SES (in models assessing the effect of social vulnerabilities) only slightly attenuated associations.

Individual components of MetS

Results of effects socioeconomic disadvantages can have on individual components of MetS [WC, BP (mean of SBP and DBP), blood lipids (mean of TG and inverse HDL levels) and IR (homeostasis model assessment, HOMA-IR)] can be found in supplementary material (Table S2-S4). Out of the social disadvantages investigated, parental education and employment status revealed the strongest associations with MetS components. Associations were mainly found for HOMA-IR, SBP and WC. 


\section{Discussion}

This prospective study indicates early childhood socioeconomic disadvantages are associated with a higher metabolic risk in children. Particularly, children from non-traditional families at baseline and follow-up, children whose parents were unemployed at baseline and follow-up and children who accumulated more than 3 socioeconomic disadvantages showed a significantly higher MetS score compared to non-disadvantaged groups. These relations were independent of children's well-being score and lifestyle factors (fruit and vegetables consumption, PA, ST), although adjustment for lifestyle factors attenuated associations. Children from low-educated and low-income families at baseline also had a higher metabolic risk at T0. However, these results were no longer significant after adjusting for children's well-being score and lifestyle factors. This suggests education and income act as causes-ofcauses, i.e. SES seems to impact on well-being and lifestyle which further affects the metabolic risk.

While some studies have investigated the effect that early socioeconomic disadvantages can have on childhood MetS, to our knowledge the effect that the patterns of disadvantages over time and the accumulation of disadvantages can have on MetS risk score and every single component of the metabolic risk have not been investigated yet. Our analyses allowed to assess prospectively the consequences that early socioeconomic disadvantages could have. While MetS risk score is higher in children from lowincome families, non-traditional families, children with unemployed parents and in children with three or more disadvantages, HOMA-IR seemed to be the component most affected by these disadvantages. These results highlight that early interventions could help preventing diabetes type 2 and cardiometabolic diseases in young children.

\section{Possible mechanisms}

Some theories have sought to explain the reasons why MetS risk is more common among socioeconomically disadvantaged groups i.e. due to mental health, biological and behavioural factors.

Stress over time has been linked to obesity and MetS[24]. Disadvantaged groups might be more exposed to chronic stress due to, among others, social isolation, financial constraints and lack of social support, which could result in negative parental cognitions, behaviors and a stressful context in children's lives. Stress can produce short-term adaptive changes in metabolism turning into maladaptive when organism is under long-term stress[25]. Chronic stress increases the activity of hypothalamic-pituitary-adrenal axis and sympathetic nervous system, which activate central pathways that stimulate the release of 
glucocorticoids (i.e. cortisol) and catecholamines. Excessive and sustained cortisol secretion has been associated with all components of MetS, potentially via an acute inflammatory immune response[26,27]. Another possible explanation on how socioeconomic disadvantages may increase the risk of MetS is its association with unhealthy lifestyle behaviours including overeating, consumption of energy-dense foods, higher sedentary behaviours and lower levels of PA[11-13].

\section{Socioeconomic status: parental education and income}

Our results are consistent with previous literature indicating an inverse relationship between SES and MetS in developed and developing countries[28, 29]. Nevertheless, most studies assessing the risk factors of MetS have been conducted in adults[28-30] and only a few in children[31]. In a Canadian study, participants aged 10-18 from households with the highest SES had the lowest prevalence of one or more MetS risk factors[31]. Another study concluded lower SES in childhood could be associated with an increased risk of MetS, impaired fasting glucose and type 2 diabetes in adulthood[8]. According to this 31-year follow-up study, disadvantages in early life have negative future consequences on health. These studies have not considered key variables such as ST and well-being proven to have a potentially mediating role. In our study, associations were attenuated after adjusting for lifestyle indicators and children's well-being, above all regarding education and income. Children from low-educated and lowincome families are more likely to live in more deprived neighborhoods with lower availability of fresh products, more fast-food outlets and few recreational opportunities resulting in poorer lifestyles and wellbeing, supporting the role of these variables as causes-of-causes[32].

\section{Social network}

A low social network of parents has been found to be negatively related to different obesity-related lifestyles in children[12-14]. Children of parents with low social networks were more likely to show an eating pattern characterized by a high consumption of snacks and fast food, lower levels of PA, higher levels of ST and more psychosocial problems than children whose parents reported to have a stronger social network. Contrary to our expectations, social network was not a relevant predictor of MetS risk but results pointed to the expected direction. 
We are not aware of any papers investigating association of parent's social network and children's MetS but in adults, several studies have indicated MetS risk was higher in individuals with a lower social support, irrespective of SES[24].

\section{Family structure}

Our results showed children with a non-traditional family structure and those children whose parents did not offer any information regarding this question had a higher MetS score compared to children who were living with both biological parents. Previous investigations indicated children from non-traditional families had more psychosocial problems than children from traditional families[14, 33]. Parents in nontraditional families might have lower levels of organization, less emphasis on active-recreational pursuits and less cohesion than traditional families, which might be disruptive for children and cause higher levels of stress[34]. Moreover, risk factors of MetS such as physical inactivity and obesity seemed to be different in traditional and non-traditional families. Indeed, children from non-traditional families are less likely to participate in organized sports[35] and have higher levels of overweight/obesity[36].

\section{Migrant background}

Some studies have focused on the impact that migrant status or some ethnicities could have on the risk of MetS, showing a higher prevalence of MetS in migrants than in natives[37]. Children with a migrant background may be at a higher risk of MetS as they have low-quality diets, higher levels of ST, more psychosocial problems and higher levels of obesity than native peers[11-14]. Despite previous findings, our results did not suggest an increased risk of MetS in migrant children. Additionally, specific ethnicity including genetic factors might influence perceived stressors and behavior, which was not assessed in this study[11].

\section{Employment status}

Children with unemployed parents at baseline and two-years later had a higher risk of MetS than children whose parents were employed at both time points. This was the strongest predictor of a higher MetS in children independently of education and income. Having a job goes beyond the notion of earning a salary affecting family and children's daily life and lifestyle, self-esteem, social life, and well-being[38]. We believe this is the first work investigating the relationship between parental employment status and risk of 
MetS in children. Nevertheless, consistent with our results, one study in adults found the OR for MetS was two times higher in those unemployed compared to employed subjects[39]. Unemployment entails anxiety, distress, depression and a decrease in activity in adults[38] that might be felt by children and may worsen their lifestyle factors and mental health and subsequently their MetS risk[12-14].

\section{Accumulation of socioeconomic disadvantages}

Our findings revealed children who accumulated 3 to 6 socioeconomic disadvantages were at a higher risk of MetS than children with no disadvantages and this effect seemed to be stronger after two years. Several studies have reported a dose-response relationship between number of socioeconomic disadvantages and increased risk for cardiometabolic diseases[40], which is consistent with our result.

\section{Strengths and limitations}

We acknowledge this study has limitations. The final sample was limited to children providing blood samples at baseline and follow-up, which reduced the sample included in these analyses. Moreover, children excluded from the analysis were more likely to belong to socioeconomically disadvantages groups and to be overweight/obese than children included in the analysis. Since a substantial proportion of children did not participate in the study or were excluded from the final analysis a selection bias in this study cannot be precluded and therefore, results should be interpreted with caution. Finally, important information on health determinants such as quantity of salt and sugar intake, alcohol habits, smoking and illicit drugs was not assessed in the present study and could hence not be considered.

Major strengths of this study include: prospective design, large sample of European children of different ages including repeated blood collections; consideration of lifestyle factors and psychosocial well-being of children as mediators; standardized covariate assessment and the use of a continuous MetS risk score based on newly derived reference values. To our knowledge, no other prospective investigation has studied the impact of a set of socioeconomic disadvantages in children on the total MetS risk score.

\section{Conclusion}

Early life exposure to socioeconomic disadvantages, particularly, living in low-educated families, having a non-traditional family structure and parental unemployment, are associated with higher MetS risk during childhood. Lifestyle factors and children's well-being are significant mediators attenuating the 
association between socioeconomic disadvantages and metabolic risk. Despite the independent effect of socioeconomic disadvantages on MetS, socioeconomic disadvantages can be seen as causes-of-causes because they seem to influence children's well-being and lifestyle, which further affect metabolic risk. These results highlight the importance of focusing on children of socioeconomically disadvantaged groups in order to decrease prevalence of MetS and health inequalities in adulthood.

\section{Acknowledgements}

This work was done as part of the IDEFICS Study (http://www.idefics.eu). We gratefully acknowledge the financial support of the European Commission within the Sixth RTD Framework Programme Contract No. 016181 (FOOD). I.I has been financed by the FPU (grant reference FPU014/00922) Predoctoral Programs (Spanish Ministry of Education and Science). We thank the IDEFICS children and their parents who generously volunteered and participated in this project. The authors' contributions were as follows: I.I carried out the statistical analysis and drafted the manuscript along with N.M and C.B. C.B. designed the statistical analyses. K.B., J.M. F.-A., G.E., M.W. and P.R. developed the measurement instruments; W.A., S.M., D.M., L.R., L.A.M., M.T and T.V. supervised the national data collection. All the authors read and critically reviewed the manuscript.

\section{References}

1. World Health Organization (WHO). Cardiovascular diseases (CVDs); 2017. http://www.who.int/mediacentre/factsheets/fs317/en/. Accessed 11 November 2017.

2. Galassi A, Reynolds K, He J. Metabolic syndrome and risk of cardiovascular disease: a metaanalysis. Am J Med 2006;119:812-819.

3. Scuteri A, Laurent S, Cucca F, et al. Metabolic syndrome across Europe: different clusters of risk factors. Eur J Prev Cardiol 2015;22:486-491.

4. Ahrens W, Moreno LA, Marild S, et al. Metabolic syndrome in young children: definitions and results of the IDEFICS study. Int J Obes (Lond) 2014;38:S4-14.

5. Weiss R, Dziura J, Burgert TS, et al. Obesity and the metabolic syndrome in children and adolescents. N Engl J Med 2004;350:2362-2374.

6. Ramsay SE, Whincup PH, Morris R, Lennon L, Wannamethee SG. Is socioeconomic position related to the prevalence of metabolic syndrome?: influence of social class across the life course in a population-based study of older men. Diabetes Care 2008;31:2380-2382.

7. Pate RR, Mitchell JA, Byun W, et al. Sedentary behaviour in youth. Br J Sports Med 2011;45:906-913.

8. Puolakka E, Pahkala K, Laitinen TT, et al. Childhood Socioeconomic Status in Predicting Metabolic Syndrome and Glucose Abnormalities in Adulthood: The Cardiovascular Risk in Young Finns Study. Diabetes Care 2016;39:2311-2317. 
9. Chichlowska KL, Rose KM, Diez-Roux AV, et al. Life course socioeconomic conditions and metabolic syndrome in adults: the Atherosclerosis Risk in Communities (ARIC) Study. Ann Epidemiol 2009; 19:875-883.

10. Suglia SF, Koenen KC, Boynton-Jarrett R, et al. Childhood and Adolescent Adversity and Cardiometabolic Outcomes: A Scientific Statement From the American Heart Association. Circulation 2018;137:e15-e28.

11. Iguacel I, Fernández-Alvira JM, Labayen I, et al. Social vulnerabilities as determinants of overweight in 2-, 4- and 6-year-old Spanish children. European Journal of Public Health 2017;27:788-796.

12. Iguacel I, Fernandez-Alvira JM, Bammann K, et al. Social vulnerability as a predictor of physical activity and ST in European children. Int J Public Health 2017; 63:283-295.

13. Iguacel I, Fernandez-Alvira JM, Bammann K, De Clercq B, Eiben G, Gwozdz W, et al. Associations between social vulnerabilities and dietary patterns in European children: the Identification and prevention of Dietary- and lifestyle-induced health EFfects In Children and infantS (IDEFICS) study. Br J Nutr 2016;116:1288-1297.

14. Iguacel I, Michels N, Fernandez-Alvira JM et al. Associations between social vulnerabilities and psychosocial problems in European children. Results from the IDEFICS study. Eur Child Adolesc Psychiatry 2017;26:1105-1117.

15. De Henauw S, Verbestel V, Marild S, et al. The IDEFICS community-oriented intervention programme: a new model for childhood obesity prevention in Europe? Int J Obes (Lond) 2011;35:S16-23.

16. Ahrens W, Bammann K, Siani A, et al. The IDEFICS cohort: design, characteristics and participation in the baseline survey. Int J Obes (Lond) 2011;35:S3-15.

17. The UNESCO Institute for Statistics. International standard classification of education (ISCED); 1997. http://www.uis.unesco.org/Education/Documents/isced-2011-en.pdf. Accessed14 March 2016.

18. Alpert BS. Validation of the Welch Allyn Spot Vital Signs blood pressure device according to the ANSI/AAMI SP10: 2002. Accuracy and cost-efficiency successfully combined. Blood Press Monit 2007; 12:345-347.

19. Matthews DR, Hosker JP, Rudenski AS, et al. Homeostasis model assessment: insulin resistance and beta-cell function from fasting plasma glucose and insulin concentrations in man. Diabetologia. 1985;28:412-419.

20. Bullinger M, Brutt AL, Erhart M, et al. Psychometric properties of the KINDL-R questionnaire: results of the BELLA study. Eur Child Adolesc Psychiatry 2008;17:125-132.

21. Ravens-Sieberer U, Bullinger M. Assessing health-related quality of life in chronically ill children with the German KINDL: first psychometric and content analytical results. Qual Life Res 1998;7:399-407.

22. Huybrechts I, Bornhorst C, Pala V, et al. Evaluation of the Children's Eating Habits Questionnaire used in the IDEFICS study by relating urinary calcium and potassium to milk consumption frequencies among European children. Int J Obes (Lond) 2011;35:S69-78.

23. Kim S, Egerter S, Cubbin C, et al. Potential implications of missing income data in populationbased surveys: an example from a postpartum survey in California. Public Health Rep 2007;122:753-763.

24. Stewart-Knox BJ. Psychological underpinnings of metabolic syndrome. Proc Nutr Soc 2005;64:363-369.

25. Tamashiro KL, Sakai RR, Shively CA, et al. Chronic stress, metabolism, and metabolic syndrome. Stress 2011;14:468-474.

26. Bose M, Oliván B, Laferrère B. Stress and obesity: the role of the hypothalamic-pituitaryadrenal axis in metabolic disease. Curr Opin Endocrinol Diabetes Obes 2009;16:340-346.

27. Felger JC, Lotrich FE. Inflammatory Cytokines in Depression: Neurobiological Mechanisms and Therapeutic Implications. Neuroscience 2013;246:199-229.

28. Loucks EB, Rehkopf DH, Thurston RC, et al. Socioeconomic disparities in metabolic syndrome differ by gender: evidence from NHANES III. Ann Epidemiol 2007;17:19-26.

29. Park MJ, Yun KE, Lee GE, et al. A cross-sectional study of socioeconomic status and the metabolic syndrome in Korean adults. Ann Epidemiol. 2007;17:320-326.

30. Matthews KA, Raikkonen K, Gallo L, et al. Association between socioeconomic status and metabolic syndrome in women: testing the reserve capacity model. Health Psychol 2008;27:576583. 
31. MacPherson M, de Groh M, Loukine L, et al. Prevalence of metabolic syndrome and its risk factors in Canadian children and adolescents: Canadian Health Measures Survey Cycle 1 (20072009) and Cycle 2 (2009-2011). Health Promot Chronic Dis Prev Can 2016;36:32-40.

32. Braveman P, Gottlieb L. The Social Determinants of Health: It's Time to Consider the Causes of the Causes. Public Health Rep 2014; 129:19-31.

33. Vanaelst B, De Vriendt T, Ahrens W, et al. Prevalence of psychosomatic and emotional symptoms in European school-aged children and its relationship with childhood adversities: results from the IDEFICS study. Eur Child Adolesc Psychiatry 2012;21:253-265.

34. Overstreet S, Goins J, Chen RS, et al. Family environment and the interrelation of family structure, child behavior, and metabolic control for children with diabetes. J Pediatr Psychol 1995;20:435-447.

35. McMillan R, McIsaac M, Janssen I. Family Structure as a Correlate of Organized Sport Participation among Youth. PLoS One 2016;11:e0147403.

36. Yannakoulia M, Papanikolaou K, Hatzopoulou I, et al. Association between family divorce and children's BMI and meal patterns: the GENDAI Study. Obesity (Silver Spring). 2008;16:1382-7.

37. Misra R, Patel T, Kotha P, et al. Prevalence of diabetes, metabolic syndrome, and cardiovascular risk factors in US Asian Indians: results from a national study. J Diabetes Complications 2010;24:145-153.

38. Voss M, Nylen L, Floderus B, et al. Unemployment and early cause-specific mortality: a study based on the Swedish twin registry. Am J Public Health 2004;94:2155-61.

39. Park HS, Oh SW, Cho SI, et al. The metabolic syndrome and associated lifestyle factors among South Korean adults. Int J Epidemiol 2004;33:328-336.

40. Elsenburg LK, van Wijk KJE, Liefbroer AC, et al. Accumulation of adverse childhood events and overweight in children: A systematic review and meta-analysis. Obesity (Silver Spring). 2017;25:820-832. 
Table 1. Description of the study population, stratified by children's metabolic syndrome risk score (Mets) (at risk/not at risk) at baseline (T0) and follow-up (T1). Number of participants and percentages are shown for categorical variables and mean for the continuous variables.

Total $2401(100 \%)$

Categorical variables at baseline

Age groups
$2.0-6.0$ years
$6.0-9.9$ years
Sex of the child
Male
Female
Country
Italy
Estonia
Belgium
Sweden
Germany
Hungary
Spain

\section{SOCIOECONOMIC DISADVANTAGES}

\section{Parental income}

Low

Medium

High

Missing

Parental education (ISCED)

Low

Medium

High

Missing

Social network

Minimal

Strong

Missing

Family structure $^{\mathrm{b}}$

Non-traditional family

Traditional family

Missing

Migrant status

Migrant origin

Native

Missing

Employment status

Unemployed

Non-unemployed

Missing

Patterns of parental income

D-D

D-ND

ND-D

ND-ND

Missing

Patterns of parental education

D-D

D-ND

ND-ND

Missing

Patterns of social network

D-D

D-ND

ND-D

ND-ND

Missing

Patterns of family structure

D-D

D-ND

ND-D

ND-ND
MetS risk score at T0 MetS risk score at T1

\begin{tabular}{lcccc} 
& At risk & Not at risk & At risk & Not at risk \\
\hline $853(35.5 \%)$ & 9.8 & 90.2 & 9.3 & 90.7 \\
$1548(64.5 \%)$ & 10.1 & 89.9 & 10.7 & 89.3 \\
& & & & \\
$1257(52.4 \%)$ & 10.3 & 89.7 & 10.7 & 89.3 \\
$1144(47.6 \%)$ & 9.7 & 90.3 & 9.5 & 90.5 \\
& & & & \\
$343(14.3 \%)$ & 22.4 & 77.6 & 23.9 & 76.1 \\
$304(12.7 \%)$ & 9.9 & 90.1 & 6.2 & 93.8 \\
$99(4.1 \%)$ & 6.1 & 93.9 & 5.1 & 94.9 \\
$416(17.3 \%)$ & 2.6 & 97.4 & 3.4 & 96.6 \\
$201(8.4 \%)$ & 3.5 & 96.5 & 2.0 & 98.0 \\
$552(23 \%)$ & 12.7 & 87.3 & 14.3 & 85.7 \\
$486(20.2 \%)$ & 8.0 & 92.0 & 8.4 & 91.6
\end{tabular}

\begin{tabular}{|c|c|c|c|c|}
\hline $634(26.4 \%)$ & 17.0 & 83.0 & 17.4 & 82.6 \\
\hline $696(29.0 \%)$ & 8.2 & 91.8 & 8.6 & 91.4 \\
\hline $974(40.6 \%)$ & 6.9 & 93.1 & 6.3 & 93.7 \\
\hline $97(4.0 \%)$ & 8.2 & 91.8 & 13.4 & 86.6 \\
\hline $134(5.6 \%)$ & 22.4 & 77.6 & 22.4 & 77.6 \\
\hline $1037(43.2 \%)$ & 12.5 & 87.5 & 13.3 & 86.7 \\
\hline $1227(51.1 \%)$ & 6.5 & 93.5 & 6.2 & 93.8 \\
\hline $3(0.1 \%)$ & 0.0 & 100.0 & 0.0 & 100.0 \\
\hline $257(10.7 \%)$ & 10.9 & 89.1 & 13.2 & 86.8 \\
\hline $2130(88.7 \%)$ & 9.9 & 90.1 & 9.8 & 90.2 \\
\hline $14(0.6 \%)$ & 14.3 & 85.7 & 14.3 & 85.7 \\
\hline $414(17.2 \%)$ & 11.4 & 88.6 & 13.3 & 86.7 \\
\hline $1973(82.2 \%)$ & 9.8 & 90.2 & 9.5 & 90.5 \\
\hline $14(0.6 \%)$ & 0.0 & 100.0 & 14.3 & 85.7 \\
\hline $235(9.8 \%)$ & 8.9 & 91.1 & 10.6 & 84.4 \\
\hline $2160(90.0 \%)$ & 10.1 & 89.9 & 10.1 & 89.9 \\
\hline $6(0.2 \%)$ & 0.0 & 100.0 & 16.7 & 83.3 \\
\hline $130(5.4 \%)$ & 12.3 & 87.7 & 13.8 & 86.2 \\
\hline $2228(92.8 \%)$ & 9.6 & 90.4 & 9.8 & 90.2 \\
\hline $43(1.8 \%)$ & 20.9 & 79.1 & 16.3 & 83.7 \\
\hline $384(16.0 \%)$ & 16.7 & 83.3 & 17.2 & 82.8 \\
\hline $158(6.6 \%)$ & 14.6 & 85.4 & 11.4 & 88.6 \\
\hline $116(4.8 \%)$ & 15.5 & 84.5 & 12.1 & 87.9 \\
\hline $1388(57.8 \%)$ & 6.8 & 93.2 & 6.8 & 93.2 \\
\hline $355(14.8 \%)$ & 11.5 & 88.5 & 14.6 & 85.4 \\
\hline $111(4.6 \%)$ & 20.7 & 79.3 & 19.8 & 80.2 \\
\hline $35(4.5 \%)$ & 25.7 & 74.3 & 28.6 & 71.4 \\
\hline $2094(87.2 \%)$ & 9.0 & 91.0 & 9.1 & 90.9 \\
\hline $161(6.7 \%)$ & 11.8 & 88.2 & 13.7 & 86.3 \\
\hline $106(4.4 \%)$ & 8.5 & 91.5 & 11.3 & 88.7 \\
\hline $131(5.5 \%)$ & 12.2 & 87.8 & 14.5 & 85.5 \\
\hline $131(5.5 \%)$ & 10.7 & 89.3 & 9.9 & 90.1 \\
\hline $1858(77.4 \%)$ & 9.8 & 90.2 & 9.5 & 90.5 \\
\hline $175(7.3 \%)$ & 10.9 & 89.1 & 13.1 & 86.9 \\
\hline $266(11.1 \%)$ & 10.2 & 89.8 & 13.5 & 86.5 \\
\hline $107(4.5 \%)$ & 12.1 & 87.9 & 12.1 & 87.9 \\
\hline $102(4.2 \%)$ & 15.7 & 81.4 & 18.6 & 81.4 \\
\hline $1757(73.2 \%)$ & 9.4 & 90.6 & 8.8 & 91.2 \\
\hline
\end{tabular}




\begin{tabular}{|c|c|c|c|c|c|}
\hline Missing & $169(7.0 \%)$ & 10.7 & 89.3 & 13.0 & 87.0 \\
\hline \multicolumn{6}{|l|}{ Patterns of employment } \\
\hline D-D & $47(2 \%)$ & 14.9 & 85.1 & 17.0 & 83.0 \\
\hline D-ND & $65(2.7 \%)$ & 10.8 & 89.2 & 10.8 & 89.2 \\
\hline ND-D & $156(6.5 \%)$ & 14.7 & 85.3 & 12.2 & 87.8 \\
\hline ND-ND & $1885(78.5 \%)$ & 8.8 & 91.2 & 9.2 & 90.8 \\
\hline Missing & $248(10.3 \%)$ & 14.9 & 85.1 & 14.9 & 85.1 \\
\hline \multicolumn{6}{|c|}{ Number of socioeconomic disadvantages ${ }^{c}$} \\
\hline 3-6 disadvantages & $138(5.7 \%)$ & 18.1 & 81.9 & 21.7 & 79.3 \\
\hline 2 disadvantages & $273(11.4 \%)$ & 12.5 & 87.5 & 11.4 & 88.6 \\
\hline 1 disadvantage & $571(23.8 \%)$ & 12.6 & 87.4 & 11.6 & 88.4 \\
\hline 0 disadvantages & $1172(48.8 \%)$ & 6.9 & 93.1 & 6.7 & 93.3 \\
\hline Missing & $247(10.3 \%)$ & 11.3 & 88.7 & 15.8 & 84.2 \\
\hline \multicolumn{6}{|c|}{ CONTINUOUS VARIABLES (MEAN, SD) } \\
\hline Fruit-vegetables [times/week] & $18.0(10.8)$ & $17.3(10.7)$ & $18.1(12.4)$ & $17.2(11.4)$ & $18.1(10.8)$ \\
\hline Physical Activity [h/week] & $18.5(10.2)$ & $18.5(11.3)$ & $18.5(10.0)$ & $19.4(12.6)$ & $18.4(9.9)$ \\
\hline Total screen time $[\mathrm{h} /$ week] & $11.6(6.9)$ & $13.1(8.6)$ & $11.4(6.7)$ & $13.5(8.4)$ & $11.4(6.7)$ \\
\hline Well-being score ${ }^{\mathrm{d}}$ & $40.1(4.4)$ & $39.4(4.6)$ & $40.2(4.4)$ & $39.2(4.6)$ & $40.2(4.5)$ \\
\hline
\end{tabular}

SD Standard Deviation, $D-D$ Disadvantaged at T0 and T1, ND-D Non-Disadvantaged at T0 and Disadvantaged at T1, ND-ND NonDisadvantaged at $\mathrm{T} 0$ and $\mathrm{T} 1$.

${ }^{a}$ Social network was assessed with the question how many persons they could rely on in case of need: minimal ( $0-1$ person) and strong (2 persons).

${ }^{\mathrm{b}}$ Family structure: If the child did not live with both biological parents, the family was defined as a 'non-traditional family'.

${ }^{\mathrm{c}}$ A total score was calculated by adding up the scores (1 vs 0 ) of the six disadvantages (low education, low income, minimal social network, non-traditional family, migrant background, unemployed). Total score ranges from 0 (the child has none of the six disadvantages) to six (the child has all six disadvantages).

${ }^{\mathrm{d}}$ The range of the well-being score was $12-48$, the higher values indicating better well-being. 
Table 2. Associations between socioeconomic disadvantages at baseline and children's metabolic syndrome risk score (MetS) at T0 (baseline) and T1 (follow-up). Results from linear mixed-effects models: $\beta$ estimates, 99\% confidence intervals $(\mathrm{CI})$ and p-values are shown.

\begin{tabular}{|c|c|c|c|c|c|c|c|c|c|c|c|c|}
\hline & \multicolumn{6}{|c|}{ METS RISK SCORE AT T0 } & \multicolumn{6}{|c|}{ METS RISK SCORE AT T1 } \\
\hline & \multicolumn{3}{|c|}{ Basic Adjustment $^{\mathrm{a}}$} & \multicolumn{3}{|c|}{ Full Adjustment $^{\mathrm{b}}$} & \multicolumn{3}{|c|}{ Basic Adjustment $^{\mathrm{c}}$} & \multicolumn{3}{|c|}{ Full Adjustment $^{\mathrm{d}}$} \\
\hline & $\boldsymbol{\beta}$ & $99 \% \mathrm{CI}$ & p-value & $\boldsymbol{\beta}$ & $99 \% \mathrm{CI}$ & p-value & $\boldsymbol{\beta}$ & $99 \% \mathrm{CI}$ & p-value & $\boldsymbol{\beta}$ & $99 \% \mathrm{CI}$ & p-value \\
\hline \multicolumn{13}{|l|}{ Parental education } \\
\hline High (Reference) & 0.00 & & & 0.00 & & & 0.00 & & & 0.00 & & \\
\hline Low & 0.28 & $0.04-0.52$ & 0.002 & 0.24 & $-0.03-0.48$ & 0.011 & 0.15 & $-0.02-0.32$ & 0.021 & 0.13 & $-0.04-0.30$ & 0.050 \\
\hline Medium & 0.17 & $0.06-0.28$ & 0.000 & 0.15 & $0.04-0.26$ & 0.000 & 0.07 & $-0.09-0.15$ & 0.022 & 0.06 & $-0.02-0.14$ & 0.059 \\
\hline Missing & -0.08 & $-1.54-1.37$ & 0.878 & -0.19 & $-1.66-1.28$ & 0.734 & -0.48 & $-1.53-0.57$ & 0.237 & -0.55 & $-1.61-0.51$ & 0.179 \\
\hline \multicolumn{13}{|l|}{ Parental income } \\
\hline High (Reference) & 0.00 & & & 0.00 & & & 0.00 & & & 0.00 & & \\
\hline Low & 0.17 & $0.01-0.31$ & 0.004 & 0.14 & $-0.01-0.29$ & 0.017 & 0.06 & $-0.04-0.16$ & 0.166 & 0.05 & $-0.03-0.48$ & 0.251 \\
\hline Medium & 0.09 & $-0.03-0.21$ & 0.072 & 0.07 & $-0.05-0.20$ & 0.142 & 0.01 & $-0.07-0.10$ & 0.706 & 0.01 & $0.04-0.26$ & 0.827 \\
\hline Missing & 0.05 & $-0.26-0.27$ & 0.958 & -0.04 & $-0.30-0.21$ & 0.917 & & $-0.20-0.17$ & 0.805 & -0.02 & $-0.21-0.17$ & 0.741 \\
\hline \multicolumn{13}{|l|}{ Social network ${ }^{\mathrm{e}, \mathrm{f}}$} \\
\hline Strong (Reference) & 0.00 & & & & & & 0.00 & & & 0.00 & & \\
\hline Minimal & -0.03 & $-0.25-0.20$ & 0.724 & -0.12 & $-0.29-0.04$ & 0.057 & 0.07 & $-0.06-0.18$ & 0.131 & 0.07 & $-0.04-0.19$ & 0.129 \\
\hline Missing & 0.37 & $-0.02-0.76$ & 0.015 & 0.12 & $-0.54-0.79$ & 0.630 & -0.05 & $-0.53-0.43$ & 0.781 & 0.03 & $-0.51-0.43$ & 0.816 \\
\hline \multicolumn{13}{|l|}{ Family structure ${ }^{\mathrm{e}, \mathrm{g}}$} \\
\hline Traditional (Reference) & 0.00 & & & & & & 0.00 & & & & & \\
\hline Non-traditional & -0.05 & $-0.18-0.09$ & 0.365 & -0.05 & $-0.19-.0 .08$ & 0.315 & 0.10 & $-0.00-0.20$ & 0.010 & 0.10 & $-0.00-.0 .20$ & 0.013 \\
\hline Missing & -0.15 & $-0.81-0.50$ & 0.539 & -0.16 & $-0.82-0.49$ & 0.518 & 0.23 & $-0.23-0.70$ & 0.196 & 0.23 & $-0.23-0.70$ & 0.202 \\
\hline \multicolumn{13}{|l|}{ Migrant status $^{\mathrm{e}}$} \\
\hline Native (Reference) & 0.00 & & & & & & 0.00 & & & 0.00 & & \\
\hline Migrant origin & 0.02 & $-0.15-0.19$ & & 0.01 & $-0.17-0.18$ & 0.969 & 0.00 & $-0.14-0.14$ & 0.670 & -0.02 & $-0.15-0.10$ & 0.583 \\
\hline Missing & 0.24 & $-0.76-1.24$ & & 0.27 & $-0.73-1.27$ & 0.483 & 0.34 & $-0.38-1.05$ & 0.226 & 0.36 & $-0.35-1.08$ & 0.190 \\
\hline \multicolumn{13}{|l|}{ Employment status $^{\mathrm{e}}$} \\
\hline Employed (Reference) & 0.00 & & & 0.00 & & & 0.00 & & & 0.00 & & \\
\hline Unemployed & -0.03 & $-0.25-0.20$ & 0.724 & -0.03 & $-0.26-0.20$ & 0.705 & 0.12 & $-0.04-0.28$ & 0.063 & 0.12 & $0.06-1.95$ & 0.051 \\
\hline Missing & 0.37 & $-0.02-0.76$ & 0.015 & 0.35 & $-0.03-0.74$ & 0.020 & 0.06 & $-0.21-0.34$ & 0.566 & 0.06 & $-0.22-0.34$ & 0.590 \\
\hline
\end{tabular}

Statistically significant results considering $99 \%$ CI are shown in bold font.

All models include random effects (school/kindergarten, country) to account for the study design.

${ }^{a}$ Basic models at T0 were adjusted for baseline age and sex.

${ }^{\mathrm{b}}$ Full models at T0 were additionally adjusted for well-being score and lifestyle indicators (frequency of fruit and vegetable consumption, physical activity, total screen time) at baseline.

${ }^{\mathrm{c}}$ Basic models at T1 were adjusted for baseline age, sex, study region (intervention v. control) and children's metabolic risk syndrome score at T0.

${ }^{\mathrm{d}}$ Full models at T1 were additionally adjusted for well-being score and lifestyle indicators: frequency of fruit and vegetable consumption, physical activity, total screen time and well-being score at baseline.

e Additionally adjusted for classical SES indicators (parental education and income) in basic and full models at T0 and T1.

${ }^{\mathrm{f}}$ Social network was assessed with the question how many persons they could rely on in case of need including their family: minimal (0-1 person) and strong (>2 persons).

${ }^{\mathrm{g}}$ Family structure: If the child did not live with both his/her parents, the family was defined as a 'non-traditional family'. 
Table 3. Longitudinal associations between patterns of socioeconomic disadvantages from T0 (baseline) to T1 (follow-up) and children's metabolic syndrome (MetS) risk score at T1. Results from linear mixedeffects models: $\beta$ estimates, $99 \%$ confidence intervals (CI) and p-values are shown.

\section{SOCIOECONOMIC DISADVANTAGES OVER TIME}

\section{Parental income}

ND-ND (Reference)

D-D

D-ND

ND-D

Missing

\section{Parental education}

ND-ND (Reference)

D-D

D-ND

Missing

\section{Social network ${ }^{\mathrm{b}, \mathrm{c}}$}

ND-ND (Reference)

$\mathrm{D}-\mathrm{D}$

D-ND

ND-D

Missing

\section{Family structure ${ }^{\text {b,d }}$}

ND-ND (Reference)

D-D

D-ND

ND-D

Missing

\section{Employment status}

ND-ND (Reference)

D-D

D-ND

ND-D

Missing

\begin{tabular}{lll}
\multicolumn{3}{l}{ METS RISK SCORE AT T1 } \\
a & 99\% CI & p-value \\
0.00 & & \\
0.06 & $-0.07-0.18$ & 0.248 \\
-0.07 & $-0.22-0.08$ & 0.252 \\
0.11 & $-0.06-0.28$ & 0.112 \\
0.05 & $-0.06-0.16$ & 0.271
\end{tabular}

0.00

$0.07 \quad-0.11-0.25 \quad 0.342$

$\begin{array}{lll}0.28 & -0.02-0.58 & 0.016\end{array}$

$\begin{array}{lll}0.05 & -0.09-0.20 & 0.366\end{array}$

$\begin{array}{lll}0.00 & & \\ 0.10 & -0.08-0.28 & 0.152 \\ 0.05 & -0.10-0.21 & 0.395 \\ -0.01 & -0.17-0.15 & 0.851 \\ 0.10 & -0.04-0.25 & 0.071\end{array}$

$D-D$ Disadvantaged at T0 and T1, $D-N D$ Disadvantaged at T0 and Non-Disadvantaged at T1, ND-D Non-Disadvantaged at T0 and Disadvantaged at T1, ND-ND Non-Disadvantaged at T0 and T1

Statistically significant results considering $99 \% \mathrm{CI}$ are shown in bold font.

All models include random effects (school/kindergarten and country) to account for the study design. 
${ }^{a}$ Models were adjusted for baseline age, sex, study region (intervention v. control), children's metabolic risk syndrome at baseline, well-being score and lifestyle indicators at baseline: frequency of fruit and vegetable consumption, physical activity and total screen time.

${ }^{\mathrm{b}}$ Additionally adjusted for classical SES indicators (parental education and income).

${ }^{\mathrm{c}}$ Social network was assessed with the question how many persons they could rely on in case of need including their family: minimal (0-1 person) and strong (>2 persons).

'Family structure: If the child did not live with both his/her parents, the family was defined as a 'non-traditional family'. 


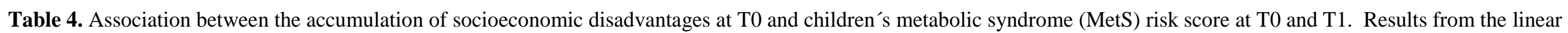
mixed-effects models: $\beta$ estimates, 99\% confidence intervals (CI) and p-values are shown.

\section{ACCUMULATION OF SOCIOECONOMIC \\ DISADVANTAGES AT TO}

Number of socioeconomic disadvantages at $\mathrm{T}^{\mathrm{c}}$

0 disadvantages (Reference)

3-6 disadvantages

2 disadvantages

1 disadvantage

Missing

\begin{tabular}{lll}
\hline \multicolumn{3}{l}{ METS RISK SCORE AT T } \\
\hline $\boldsymbol{\beta}$ & $\mathbf{9 9 \%}$ CI & p-value \\
& & \\
0.00 & & \\
0.18 & $-0.05-0.41$ & 0.052 \\
-0.10 & $-0.28-0.07$ & 0.128 \\
0.01 & $-0.11-0.14$ & 0.770 \\
0.05 & $-0.12-0.22$ & 0.474
\end{tabular}

METS RISK SCORE AT T1

99\% CI p-value

0.00

$0.21 \quad 0.04-0.37 \quad \mathbf{0 . 0 0 1}$

$0.02 \quad-0.10-0.15 \quad 0.668$

$0.03 \quad-0.05-0.12 \quad 0.358$

$0.08 \quad-0.04-0.21 \quad 0.091$

All models include random effects (school/kindergarten and country) to account for the study design.

Statistically significant results considering $99 \% \mathrm{CI}$ are shown in bold font.

${ }^{a}$ Models at T0 were adjusted for baseline age, sex, well-being score and lifestyle indicators: frequency of fruit and vegetable consumption, physical activity, total screen time and well-being score at baseline.

${ }^{\mathrm{b}}$ Models at T1 were additionally adjusted for study region (intervention v. control) and children's metabolic risk syndrome at T0.

${ }^{\mathrm{c}}$ A total score was calculated by adding up the scores ( $1 \mathrm{vs} 0$ ) of the six disadvantages (low education, low income, minimal social network, non-traditional family, migrant background, unemployed). Total score ranges from 0 (the child has none of the six disadvantages) to six (the child has all six disadvantages). 


\section{Highlights}

- Disadvantages in childhood were related to higher metabolic syndrome (MetS) risk

- Non-traditional families and unemployment contributed most to MetS risk

- Insulin resistance, systolic blood pressure and waist circumference were affected

- Lifestyle factors and children's well-being are significant mediators 


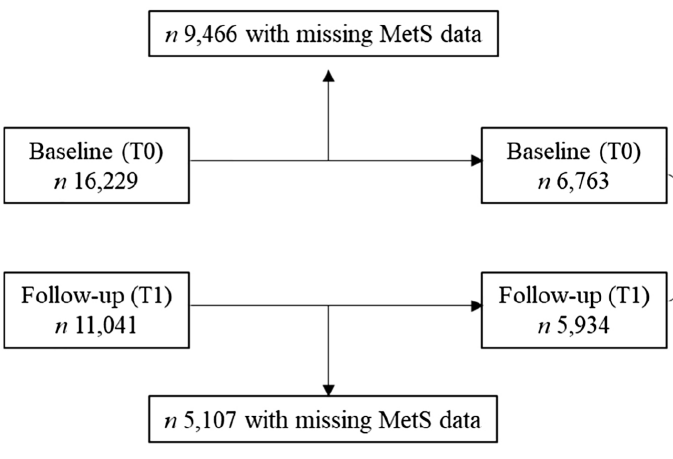

$n 533$ missing values on covariates (missing values in children's physical activity data, children's sedentary

behaviours, children's fruits and vegetables

consumption and children's well-being score)

Figure 1 